\title{
Gingival Reshaping Using Provisional Restoration in a Maxillary Anterior Implant - A Case Report
}

\author{
Karki $\mathrm{S}^{1}$, Thapa $\mathrm{D}^{2}$, Pandey $\mathrm{N}^{3}$ \\ ${ }^{1}$ Consultant Prosthodontics, Family Dental Care, Bhaktapur, Nepal \\ ${ }^{2}$ Visiting Faculty, Deaprtment of Prosthodontics, National Academy of Medical Sciences, Kathmandu, Nepal \\ ${ }^{3}$ Lecturer, Kantipur Dental College and Hospital, Kathmandu, Nepal
}

\begin{abstract}
Oral implants are regarded as one of the the most successful and reliable treatment options for replacing missing tooth/teeth. Various studies have reported that it can be used to meet the functional as well as esthetic demands of missing tooth or teeth. The most common indication of oral implants is replacement of single or multiple teeth to support the denture. While replacing tooth in the esthetic zone, the consideration should be towards creating the right emergence profile. So, replacing tooth with implant supported prosthesis in the anterior region is more challenging as compared to the posterior. With clinical examination, radiographic investigation and treatment planning good results can be achieved. This case report highlights the placement of a single-tooth dental implant in anterior region where emergence profile was created with gingival moulding similar to that of adjacent gingiva using provisionalization.
\end{abstract}

Key words: Anterior implant; Temporization; Gingival moulding

\section{Introduction}

$\mathrm{I}^{\mathrm{n}}$ n our day to day clinical practice we encounter patients with high esthetic demand. Despite successful osseointegration of dental implants, poor esthetics of definitive restoration can result in dissatisfaction with implant supported restoration. ${ }^{1}$ Success of an esthetically pleasing restoration depends on correct implant placement, a well-designed and fabricated prosthesis which is in a harmonious relationship with the surrounding soft tissue.

Gingival architecture plays a crucial role in maintaining the pink esthetics which is governed by the soft tissue and the underlying alveolar

\section{*Corresponding Author}

Dr. Nashib Pandey

Lecturer, Department of Periodontology \& Oral Implantology, $\mathrm{KDCH}$, Basundhara, Nepal.

E-mail: nashibpandey@gmail.com bone. Defects in the soft tissue may result in poor esthetics. Temporization maintains the gingival architecture, promotes the gingival health, which ultimately leads to the better clinical outcomes and the final restoration. Preformed healing abutment cannot provide emergence profile. The soft tissue profile around a maxillary central incisor is generally triangular in shape, whereas a stock transfer abutment coping that is usually available in the market is cylindrical in shape. The discrepancy between the soft tissue profile and stock transfer abutment coping does not allow to accurately convey the soft tissue information to dental laboratory. Customized impression post may help to minimize gingival distention or collapse during the impression process. $^{2}$

This article describes a case report of a gingival contouring by fabrication of a provisional implant restoration to produce an optimal emergence profile of a definitive implant restoration. This was achieved through the use 
of customized transfer abutment technique which is simple to implement and is an accurate method of recording the gingival contours during the temporization stages.

\section{Case Presentation}

A 26-year-old female patient visited the Department of Prosthodontics, $\mathrm{KDCH}$, Basundhara with the chief complaint of "missing upper front tooth since 2 weeks". The reason for tooth loss was trauma due to road traffic accident. The medical history given by the patient was non contributory. On intraoral examination missing right upper first molar was noted.(Figure 1) Preliminary radiographic examination using intraoral periapical radiograph (IOPAR) was recorded which did not reveal any root fragment. Overall periodontal status as well as occlusion was examined. The patient was explained about the various treatment options available for replacing missing teeth, and she opted for implant supported prosthesis.

Irreversible hydrocolloid impression of upper and lower arch was made, disinfected and then poured using Type 3 Gypsum product. Cone Beam Computed Tomography (CBCT) imaging was suggested to determine the available bone width for implant placement. An endo-osseous implant (Dio tapered threaded) measuring $3.3 \mathrm{~mm}$ diameter and $10 \mathrm{~mm}$ length was planned. Antibiotic amoxicillin $500 \mathrm{mg} 3$ times daily for 7 days was started one day prior the treatment. A palatally positioned crestal incision was given and the full-thickness flap was raised. Vertical releasing incision was given (Figure 2). Following the manufacturer's protocol for implant placement an osteotomy site was prepared with the help of the surgical template. (Figure 3). Pilot drill was used to mark the indentation in the area (Figure 4), followed by $2.0 \mathrm{~mm}$ drill. Guiding pin was used to check the parallelism. Intraoral Periapical radiograph confirmed the position of implant to be placed. Tapered, threaded, rough surface implant was then placed under 30rotations per minute (speed) and primary stability was achieved at $35 \mathrm{~N}$. Cover screw was used to cover the coronal end of the fixture, followed by flap closure with 3- 0 vicryl suture for healing by primary intention (Figure5). Post-operative patients instructions were given. Acrylic teeth bonded with composite to the proximal side of adjacent teeth served as temporary restoration.

Six months later after implant placement, for second stage surgery a small crestal incision was given to approach the implant site. Cover screw was removed followed by placement of open tray impression coping for PolyVinyl Siloxane impression (Figure 6, 7, 8). The shade was recorded as that of adjacent tooth i.e. A2. Custom temporary restoration was fabricated with cold cure acrylic resin. (Figure 9, 10)

The customized abutment was placed \& radiograph was taken to confirm the seating of abutment. The temporary abutment was then torqued to $35 \mathrm{~N}$ with the help of a torque wrench. The temporary crown was then placed, the proximal contacts and occlusion verified and then cemented with the help of temporary cement. Excess cement was removed and the occlusion was verified again. After 2 weeks, patient was recalled to observe gingiva for healing, the contours were compared to the adjacent tooth. The proximal contacts and occlusion was verified again at recall visit. The (Figure 11, 12) gingival moulding with composite was done to get the esthetic outcome as that of the adjacent teeth.

The soft tissue around the implant fixture was evaluated every $2^{\text {nd }}$ week after placing the provisional implant restoration. The provisional restoration was relined and shaped to achieve the desired gingival form \& emergence profile. After allowing the gingiva to stabilize for 6 weeks, a final impression was made for definitive implant restoration. Patient decided to go for 
zirconia crown. After the definitive restoration was ready, it was tried in the patient's mouth. Minor adjustments were made chairside. At last

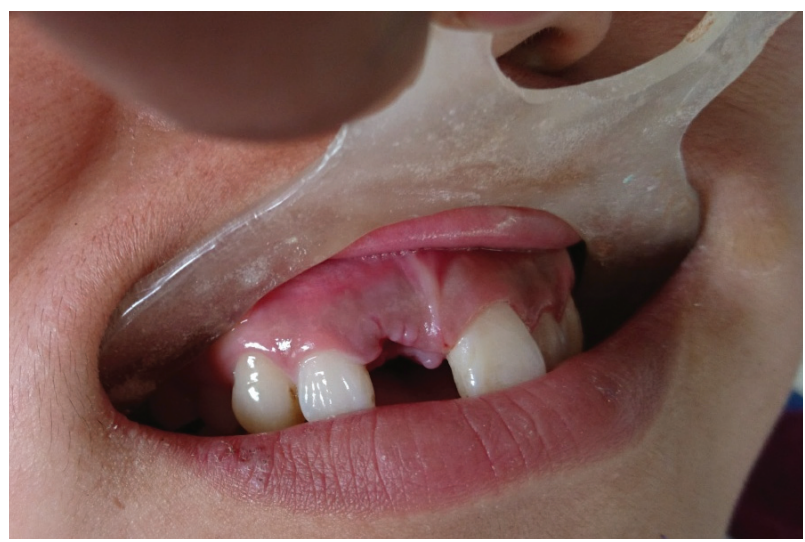

Figure 1: Missing right upper first molar

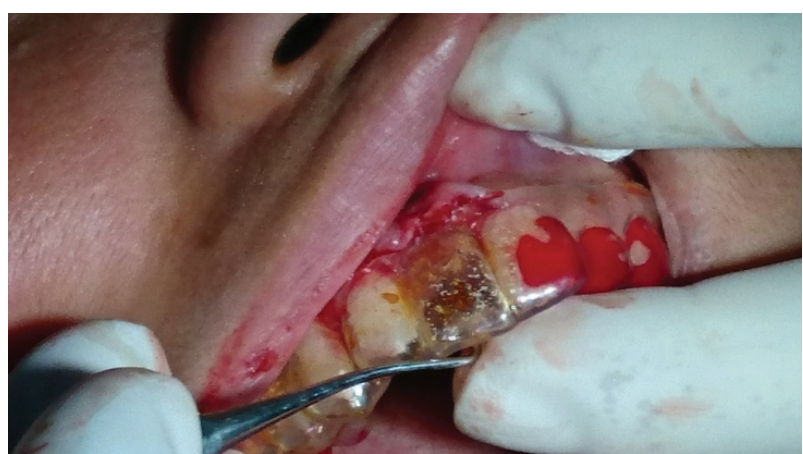

Figure 3: Surgical template a harmonious relation was obtained between the soft tissue and tooth form with the adjacent tooth (Figure 13, 14). The patient was satisfied with the final result.

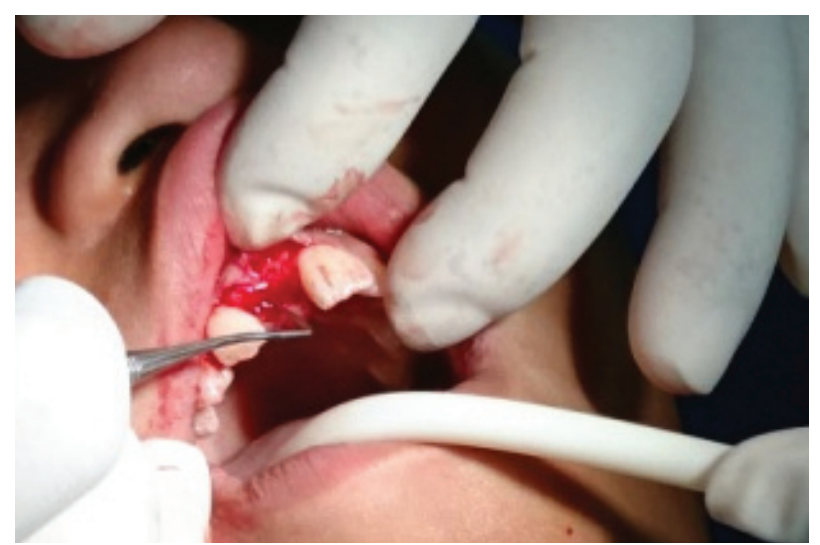

Figure 2: Vertical releasing incision

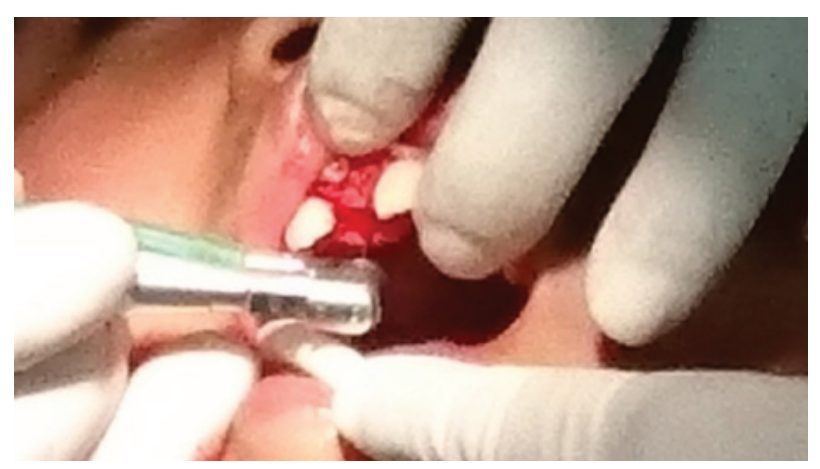

Figure 4: Pilot drill to mark indentation in the area

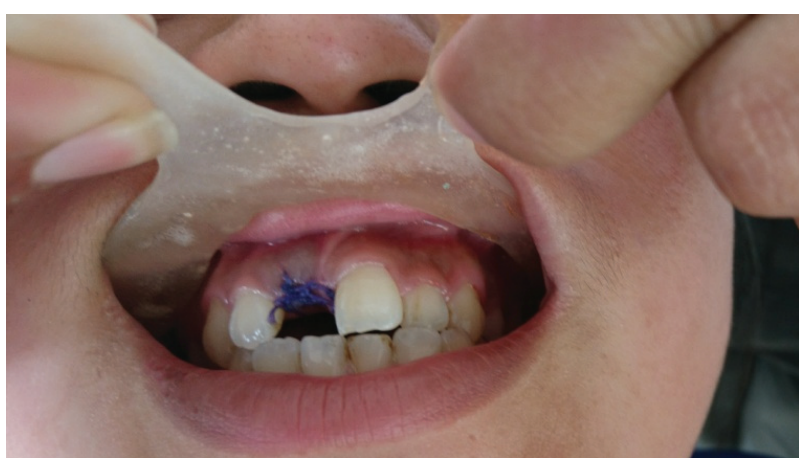

Figure 5: Flap closure with 3-0 vicryl suture 

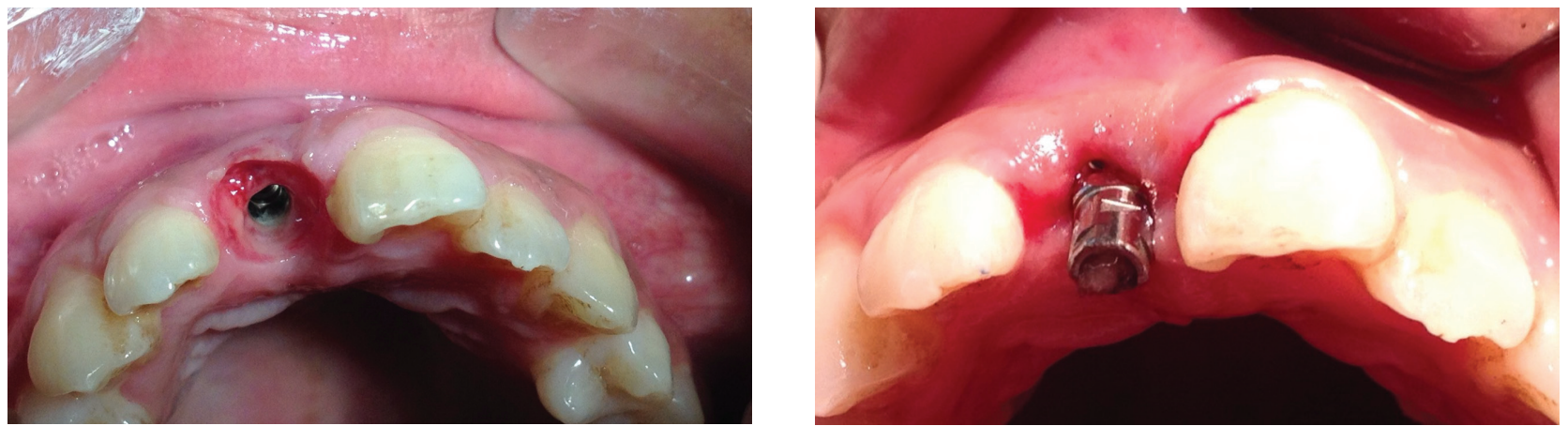

Figure 6 \& 7: Removal of cover screw followed by placement of open tray impression coping
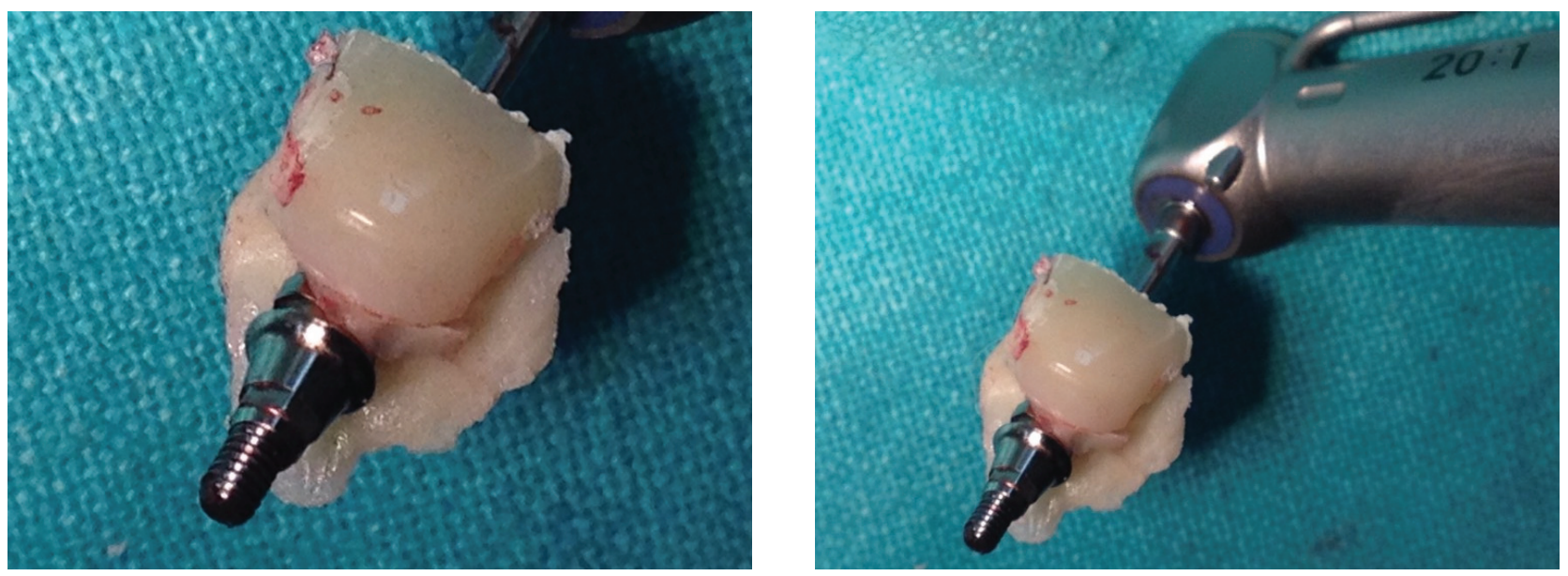

Figure 8 \& 9: Custom temporary restoration fabricated with cold cure acrylic resin
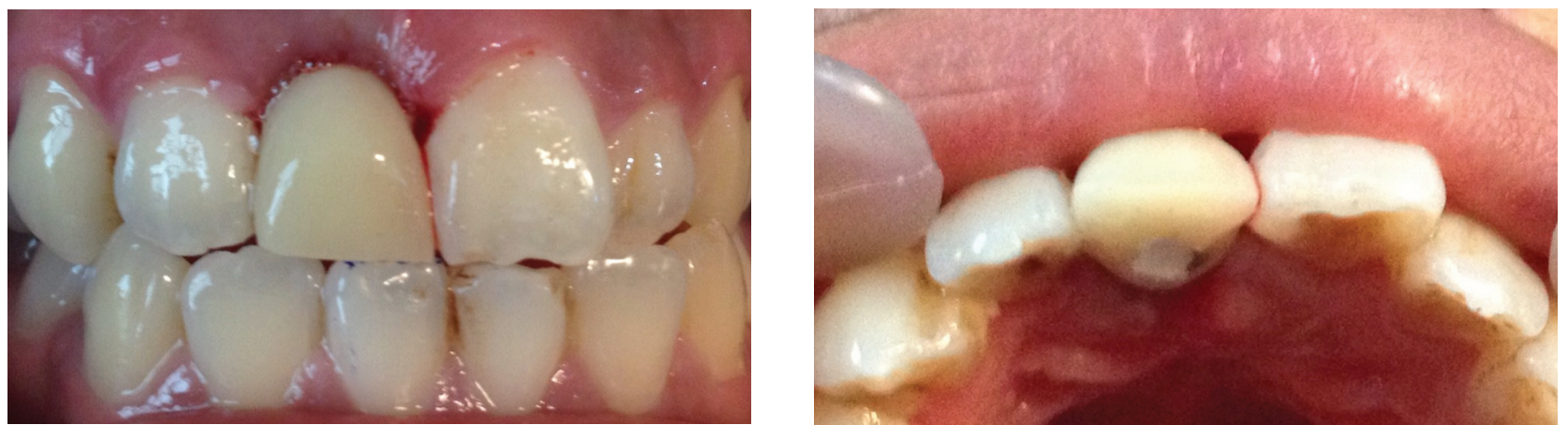

Figure 10 \& 11: Immediate positioning of temporary crown along with gingival moulding with composite
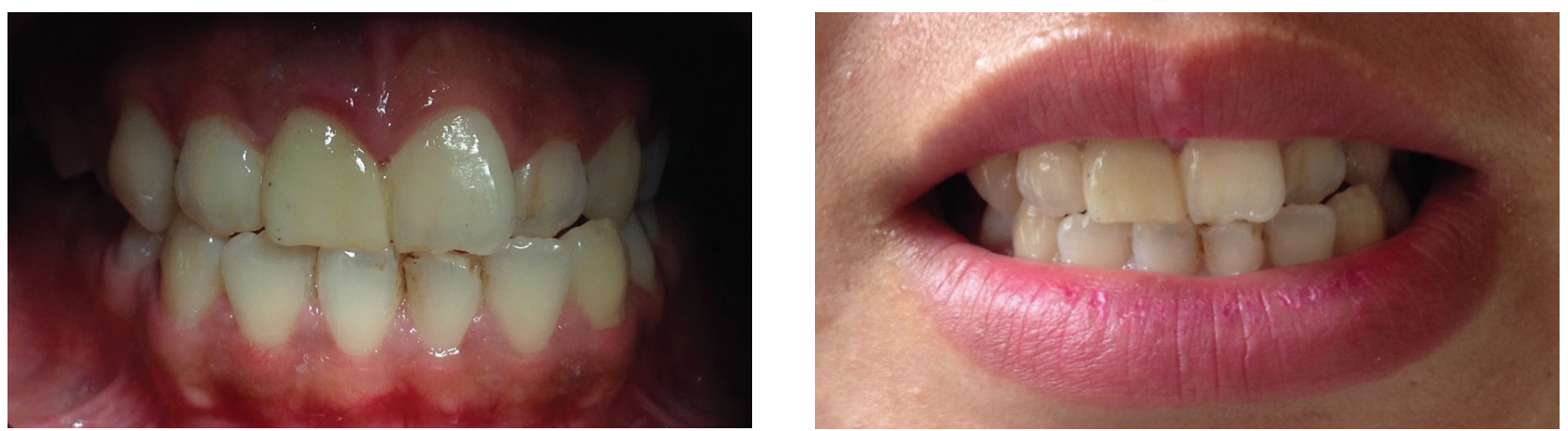

Figure 12 \& 13: Follow up after permanent restoration at 3 months 


\section{Discussion}

The anterior region of the maxilla is highly visible having great impact on the facial appearance of an individual, hence frequently termed the aesthetic zone. Single tooth replacement in this region can present many clinical challenges. Both the soft and hard tissues play a significant role .There must be harmonious relationship between the crown contour, shade, and texture along with its gingiva which must also be in harmony with the adjacent teeth.

The term "emergence profile" was first used in 1977 by Stein and Kuwata to describe tooth and crown contours as they traversed soft tissue and rose toward the contact area interproximally and height of contour facially and lingually. ${ }^{3}$ In 1990, a photographic analysis of natural teeth by Croll confirmed that most emergence profiles are relatively straight as opposed to convex or concave. ${ }^{4}$ If a restoration introduced a convexity or concavity where it didn't belong, the unnatural contour might trap plaque or otherwise disrupt the gingiva. ${ }^{5}$

The advantage served by the provisional restoration was it allowed the patient to have an idea for definitive crown as similar to the natural tooth that was present earlier. Moreover, it also helped to reform the interdental papilla between the implant crown and the natural tooth. Lightcured composite was used at the base of the provisional restoration to create an emergence profile. Reason behind this was light-curing composite resin used at the base causes less soft tissue irritation due to elimination of the auto polymerized acrylic resin monomer which was more with the acrylic resin. ${ }^{6,7}$

A screw retained restoration was preferred as it serves the purpose of better retrievability as compared to that of cement retained restoration. Furthermore, there was no excess cement which could soft tissue irritation, especially in the subgingival site. Also, a rough surface created at the crown abutment junction is also eliminated. To facilitate tissue healing the restoration was highly polished.

\section{Conclusion}

Whenever a maxillary anterior tooth is extracted, there will be more chance of collapse of the socket wall which usually occur in a bucco-palatal direction in most of the cases. In such scenario, it will be more difficult to provide a definitive restoration as it will result in compromised esthetic situation which will be challenging for dental surgeons. Reason behind it is the remaining socket may be thin or in most of the instances there will be no existence of labial plate. As a consequences, the crestal height of the socket also moves apically leaving a concavity in the labial side, adding further challenging situation. Ultimately the final restoration will be in vain as it will not be symmetrical with the surrounding structures. So, keeping in mind all these consequences, meticulous treatment planning is of utmost importance from day one i.e patient counseling is mandatory. This becomes even more important when implant has to be placed in the esthetic zone. As, more successful results are there in favor of gingival moulding through temporization which is one of the latest innovation in implantology for masking the defective site. So, this case was also done following one of the principles or guidelines for gingival molding as reported in the literature.

\section{References}

1. Varshney N, Jahan S, Praveen S, Kumar S, Rani S. Creation and Preservation of Soft Tissue Profile around Dental Implant Supported Restoration in Esthetic Zone. International Journal of Research and Reports in Dentistry 2019, 1(2), 1-7.

2. Ibraheem Fahad Alshiddi, D. Clin Dent Accurate registration of peri-implant soft tissues to create an optimal emergence profile. 
Contemp Clin Dent. 2015 Mar; 6(Suppl 1): S122-S125. doi: 10.4103/0976-237X.152967

3. Stein RS, Kuwata M. A dentist, and a dental technologist analyze current ceramo-metal procedures. Dent Clin North Am. 1977;21:729 49. [PubMed] [Google Scholar]

4. Croll BM. Emergence profiles in natural tooth contour. Part II: Clinical considerations. J Prosthet Dent. 1990; 63:374-9. [DOI: 10.1016/0022-3913(90)90223-Y] [PMID: 2184220]
5. Jameson LM, Malone WFP. Crown contours and gingival response. $\mathrm{J}$ Prosthet Dent 1982;47:620-6 [pubmed]

6. Al-Harbi SA, Edgin WA. Preservation of soft tissue contours with immediate screw-retained provisional implant crown. J Prosthet Dent 2007;98:329-332.

7. Ntounis A, Petropoulou A. A technique for managing and accurate registration of periimplant soft tissues. J Prosthet Dent 2010;104:276-279. 